\title{
4 Versorgung besonderer Wunden
}

Wunden mit viel Exsudat, besonderer Größe oder Lage stellen den Behandler vor einige Aufgaben. Hierbei sind besonders Dekubitus, Verbrennungen, das offene Abdomen und exazerbierte Tumorwunden zu nennen.

\subsection{Dekubitus}

\subsubsection{Definition und klinische Bedeutung}

Ein Dekubitus ist eine lokal begrenzte Schädigung der Haut und/oder des darunterliegenden Gewebes, meist an Stellen über knöchernen Vorsprüngen. Die Defekte entstehen durch Druck, auch in Kombination mit Scherkräften.

Ein Dekubitus gehört zu den gravierenden Gesundheitsproblemen pflegebedürftiger Patienten. Das vorhandene Wissen zeigt, dass das Auftreten eines Dekubitus weitgehend verhindert werden kann. Ausnahmen sind in pflegerisch oder medizinisch notwendigen Prioritätensetzungen, bei massiv reduziertem Gesundheitszustand oder in der selbstbestimmten, informierten Entscheidung des Patienten begründet.

Es gibt eine Reihe weiterer Faktoren, welche mit Dekubitus assoziiert werden, deren Bedeutung aber noch zu klären ist.

\subsubsection{Pathogenese}

Bestimmte Körperstellen sind besonders gefährdet, einen Dekubitus zu entwickeln. Diese Prädilektionsstellen (Kreuzbein, Fersen, Zehen, Fußknöchel, Knie, Beckenknochen, Gesäß, Wirbelvorsprünge, Schulterblatt, Ellenbogen, Ohrmuschel, Schläfenregion und der Hinterkopf, $\triangleright$ Abb. 4.1) haben nur eine geringe subkutane Weichteilschicht.

Lastet auf einer bestimmten Körperstelle zu viel Druck, werden die Haut und das darunterliegende Gewebe schlechter durchblutet. Hält der Druck länger als zwei Stunden an, kann der Sauerstoff- und Nährstoffmangel zu einem Absterben der Zellen führen. Es tritt eine Schädigung der Haut und der darunterliegenden Gewebeschichten ein.

\section{Merke}

Das Ausmaß der Schädigung ist abhängig von der Zeitdauer und der Stärke der Einwirkung. 


\subsection{Dekubitus}

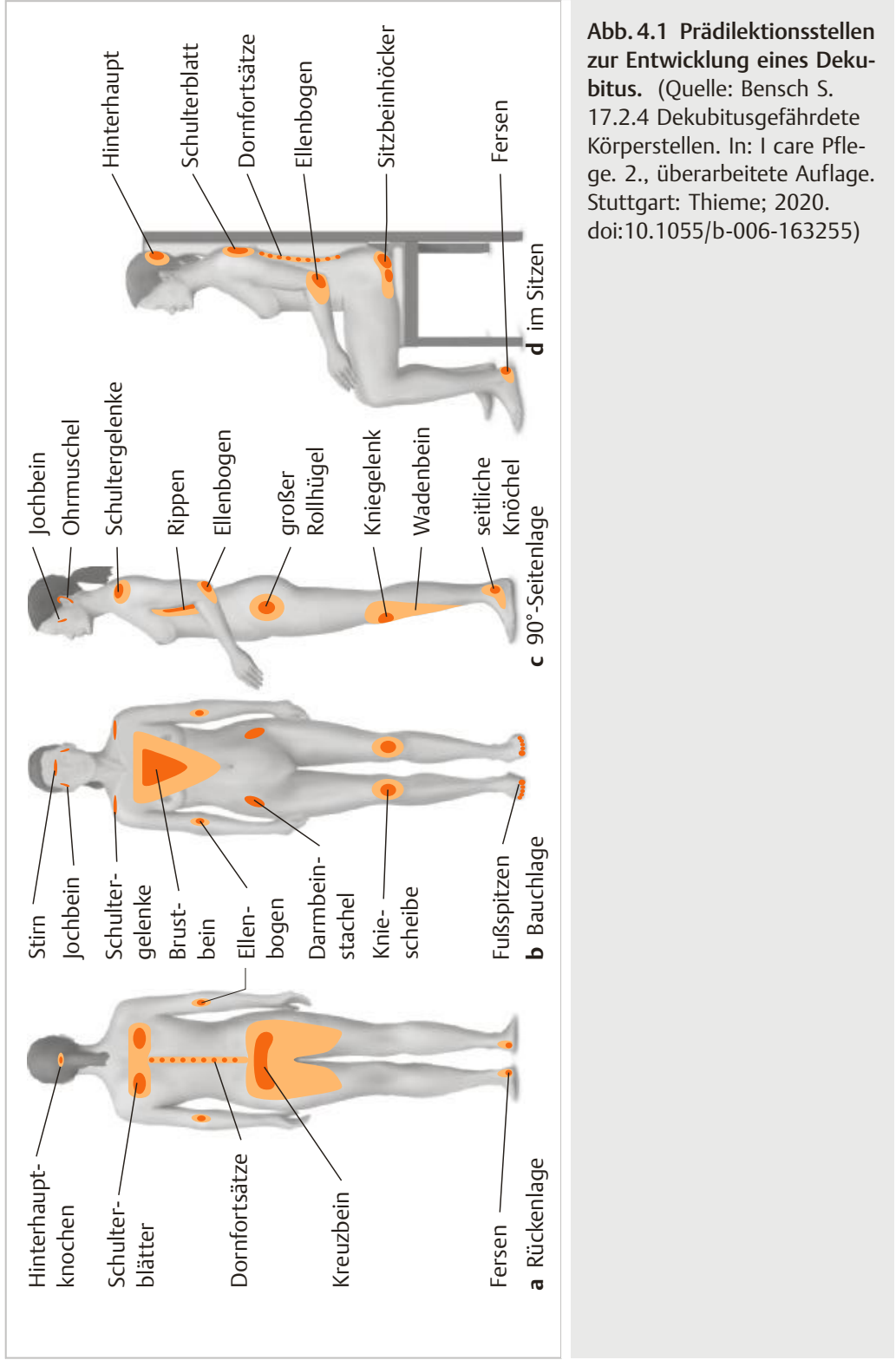


Normalerweise meldet der Körper, z. B. in Form von Schmerzen, wenn bestimmte Stellen entlastet werden müssen. Sobald wir uns dann bewegen, wird der Druck umverteilt und die Haut wieder normal durchblutet. Bei älteren und/oder nicht mehr mobilen Menschen oder bei entsprechenden fehlenden sensorischen Nervenreizen fehlt dieser Entlastungsmechanismus. Entweder können sich die Patienten nicht mehr mitteilen oder sie spüren aufgrund von Schmerzmitteln oder einer Fehlfunktion nicht, wenn der Druck zu stark wird. Dann können schon eine Falte im Bettlaken, ein Gipsverband, Fettpolster unter der Haut oder ein kurzes Abrutschen mit entsprechenden Scherkräften zu einem Dekubitus führen ( $\vee$ Abb. 4.2).

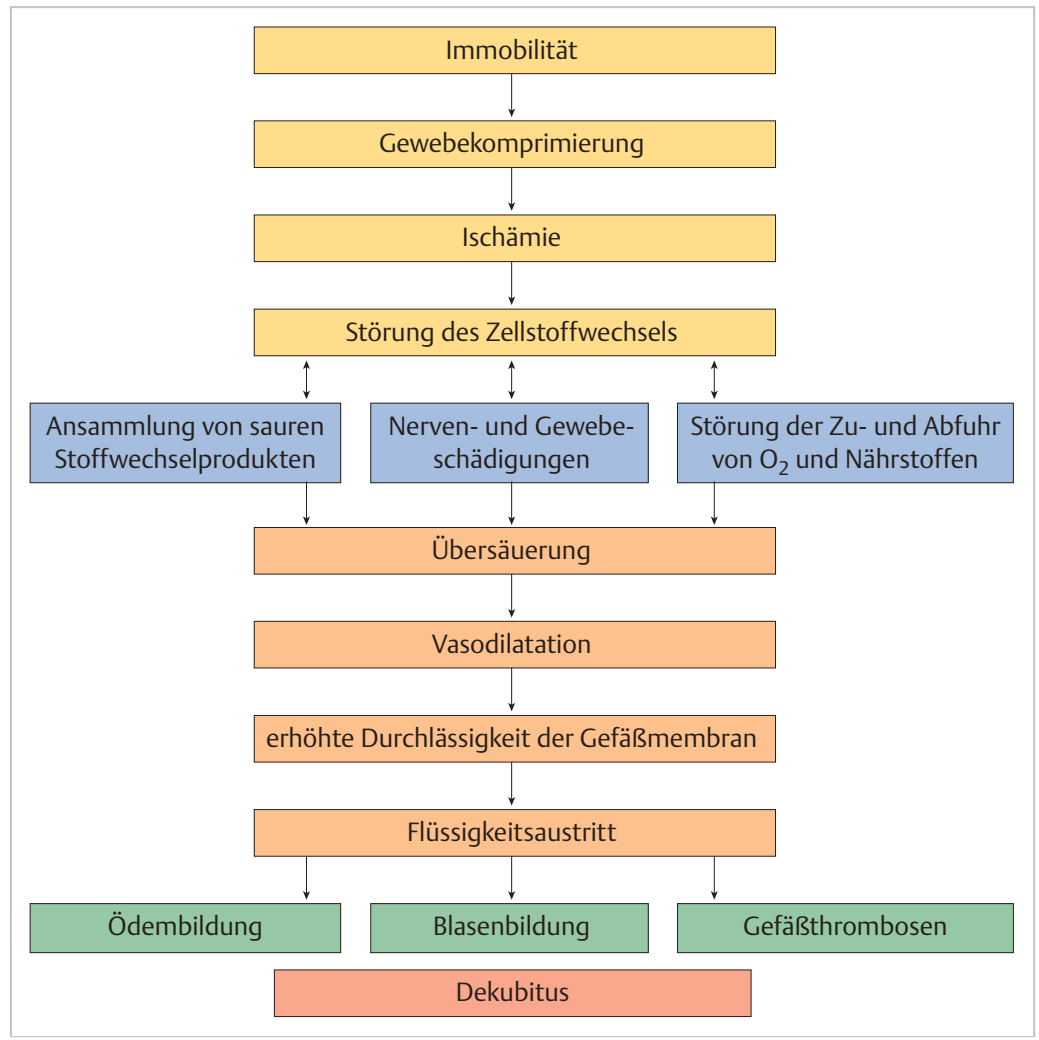

Abb.4.2 Dekubitus: Pathogenese. 
Zusätzliche Risikofaktoren:

- hohes Lebensalter

- mangelnde Beweglichkeit

- Reibung und Scherkräfte

- schlechter Hautzustand

- schlechter Ernährungszustand, z. B. Mangelernährung, Unter- oder Übergewicht

- Erkrankungen mit sensorischen Ausfällen wie z. B. Diabetes, Schlaganfall

- Medikamente

- Durchblutungsstörungen

- Inkontinenz.

\subsubsection{Risikoeinschätzung}

Eine Risikoeinschätzung für einen Dekubitus sollte am Aufnahmetag bzw. am ersten Behandlungstag unmittelbar vor Beginn der pflegerischen Tätigkeit erfolgen (und danach zu individuell festgelegten Abständen weitergeführt werden).

Es wird unterschieden zwischen

- der initialen Risikoeinschätzung und

- der differenzierten weiteren Risikoeinschätzung.

Initiale (orientierende) Risikoeinschätzung:

- Erfassung und Beobachtung des Gesundheitszustandes des Patienten - Ist der Gesundheitszustand erheblich eingeschränkt?

- Ist der Patient bereits erheblich pflegebedürftig?

- Hautinspektion einschließlich Fingertest bei vorhandener Rötung.

Differenzierte (detaillierte) Risikoeinschätzung:

- Eingeschränkte Aktivität oder Mobilität des Patienten?

- Intrinsische Risikofaktoren wie z.B. Diabetes mellitus?

- Extrinsische Risikofaktoren?

- Hautinspektion: beeinträchtigter Hautzustand/vorliegender Dekubitus?

- Durchblutungsstörungen oder eine verminderte sensorische Wahrnehmung?

- Anwendung spezifischer Assessment-Instrumente. 


\subsubsection{Therapie}

\section{Positionierung/Mobilisation}

- aktive Mobilisierung des Patienten

- ganzheitliche ununterbrochene Druckentlastung durch Freilagerung von gefährdeten Körperstellen, z. B. den Fersen mittels eines unterlegten Kissens oder Ligasano

- ganzheitliche partielle Druckentlastung durch Entlastungslagerung oder regelmäßige Mobilisation oder Positionsveränderung nach individuellem Bewegungsförderungsplan

- gewebeschonende Bewegungs-, Positionierungs- und Transfertechniken.

\section{Hilfsmittel}

- Druckverteilung durch viskoelastische Schaumstoffmatratzen, Wechseldruckmatratzen oder andere Anti-Dekubitus-Matratzen

- atmungsaktive und hautfreundliche Bettwäsche

- Bettlaken möglichst faltenfrei aufziehen

- weiche Kissen, spezielle Schaumunterlagen, Antidekubitus-Kissen im Rollstuhl.

\section{Cave}

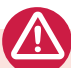

- Verzicht auf mit Wasser gefüllte Kissen oder Schläuche $\rightarrow$ fehlende Hautentlastung

- keine synthetischen oder echten Schaffelle, auch nicht als Auflagen auf druckverteilenden Matratzen

- keine Ringkissen $\rightarrow$ sie erhöhen das Dekubitus-Risiko

- auf gut sitzende Schuhe achten

- Schulung des Patienten und seiner Angehörigen.

\section{Pflege}

- Verwendung von seifenfreier, hautschonender Waschlotion und rückfettender Hautschutzsalbe

- Verzicht auf Zinksalbe, Babypuder, Vaseline, Melkfett oder Franzbranntwein 


\section{Ernährung}

- vitamin-/eiweißreiche Ernährung mit ausreichender Flüssigkeitsgabe

- bei Diabetes mellitus: Kontrollieren der Blutzuckerwerte

\section{Dekubitusprophylaxe}

Jeder dekubitusgefährdete Patient erhält eine Prophylaxe, die die Entstehung eines Dekubitus verhindern soll.

Von herausragender Bedeutung für eine erfolgreiche Prophylaxe ist die systematische Risikoeinschätzung, Information, Schulung und Beratung von Patienten/Bewohnern und ggf. den Angehörigen durch das Pflegepersonal. Dabei sind Bewegungsförderung, Druckentlastung und -verteilung sowie die Kontinuität und Evaluation prophylaktischer Maßnahmen zu gewährleisten.

\subsubsection{Differenzialdiagnosen}

Die wichtigste Differenzialdiagnose ist die Inkontinenz-assoziierte Dermatitis (IAD). Diese tritt häufig bei inkontinenten Patienten im Perianalbereich auf und ist manchmal schwer abzugrenzen (s. Kap. 4.2).

Weitere Differenzialdiagnosen sind wegdrückbare, nicht tastbare Erytheme anderer Genese sowie chronische Wunden anderer Herkunft (diabetisches Ulkus, Ulcus venosum) an für Dekubitus untypischen Prädilektionsstellen wie den Streckseiten der Extremitäten, Fußrücken oder Fingerspitzen.

\subsubsection{Internationales Klassifikationssystem für Dekubitus von NPUAP/EPUAP}

(Text übernommen und leicht gekürzt, zitiert aus National Pressure Ulcer Advisory Panel, European Pressure Ulcer Advisory Panel and Pan Pacific Pressure Injury Alliance. Prevention and Treatment of Pressure Ulcers: Quick Reference Guide. Emily Haesler (Ed.). Cambridge Media: Osborne Park, Australia; 2014)

\section{Kategorie/Stadium I: nicht wegdrückbares Erythem}

Intakte Haut mit nicht wegdrückbarer Rötung eines lokalen Bereichs gewöhnlich über einem knöchernen Vorsprung ( $\triangleright$ Abb. 4.3, $\triangleright$ Abb. 4.4). Bei dunkel pigmentierter Haut ist ein Abblassen möglicherweise nicht sichtbar, die Farbe kann sich aber von der umgebenden Haut unterscheiden. Der Bereich kann schmerzhaft, härter, weicher, wärmer oder kälter im Vergleich zum umgebenden Gewebe sein. 


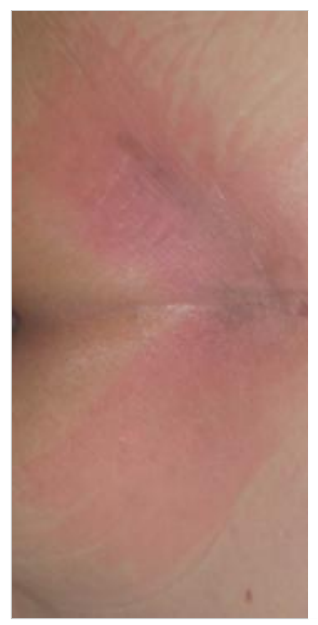

Abb. 4.3 Dekubitus Stadium I.

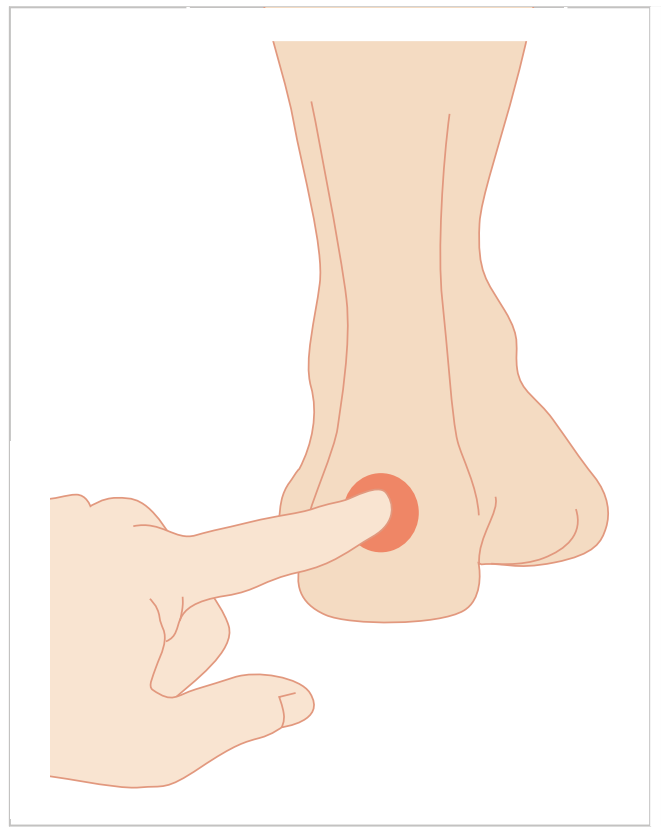

Abb. 4.4 Dekubitus

Stadium I: nicht wegdrückbares Erythem. 


\section{Kategorie/Stadium II: Teilverlust der Haut}

Teilzerstörung der Haut (bis in die Dermis/Lederhaut), die als flaches, offenes Ulkus mit einem roten bis rosafarbenen Wundbett ohne Beläge in Erscheinung tritt ( $\triangleright$ Abb. 4.5, $\triangleright$ Abb. 4.6). Kann sich auch als intakte oder offene/ruptierte, serumgefüllte Blase darstellen.

Manifestiert sich als glänzendes oder trockenes, flaches Ulkus ohne Beläge oder Bluterguss (eine livide Verfärbung weist auf eine tiefe Gewebeschädigung hin).

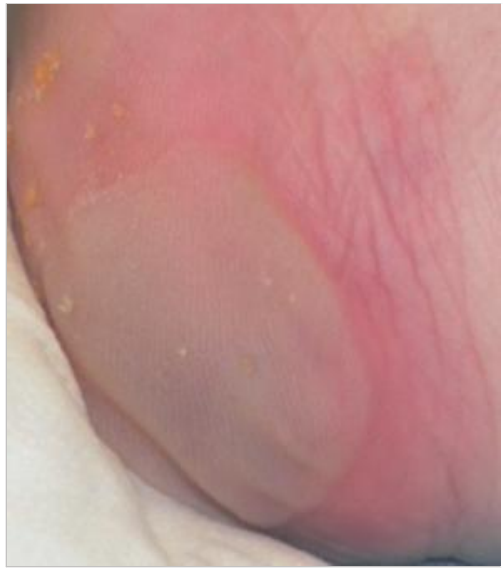

Abb. 4.5 Dekubitus Stadium II.

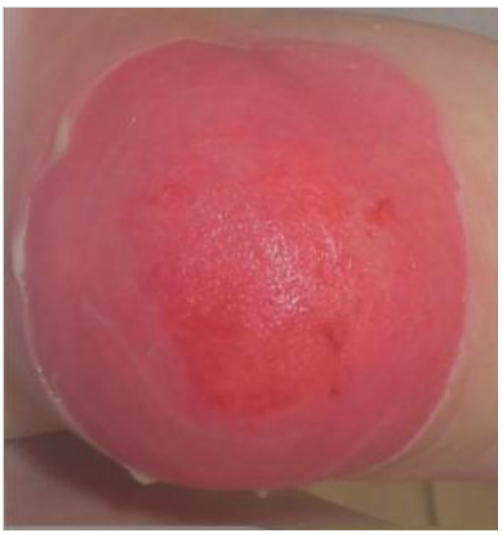

Abb. 4.6 Dekubitus Stadium II. 


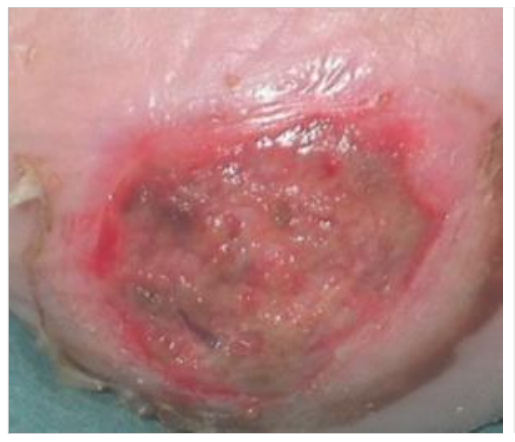

Abb. 4.7 Dekubitus Stadium III.

Diese Kategorie sollte nicht benutzt werden, um Skin Tears (Gewebezerreißungen), Verbands- oder pflasterbedingte Hautschädigungen, IAD, perineale Dermatitis, Mazerationen oder Exkoriation zu beschreiben.

\section{Kategorie/Stadium III: vollständiger Verlust der Haut}

Vollständiger Verlust von Epidermis und Dermis ( $\vee$ Abb. 4.7). Subkutanes Fett kann sichtbar sein, aber Knochen, Sehne oder Muskel liegen nicht offen. Partiell können Beläge vorhanden sein, die aber nicht die Tiefe des Gewebeverlustes verdecken. Es können Taschenbildungen oder Unterminierungen vorliegen.

Die Tiefe eines Dekubitus der Kategorie III kann je nach anatomischer Lokalisation variieren. Der Nasenrücken, das Ohr, das Hinterhaupt und der Knöchel haben kein subkutanes Gewebe und Ulzera der Kategorie III können dort relativ oberflächlich sein. Im Gegensatz dazu können besonders adipöse Bereiche einen extrem tiefen Dekubitus der Kategorie III entwickeln. Knochen oder Sehnen sind nicht sichtbar oder direkt tastbar.

\section{Kategorie/Stadium IV: vollständiger Gewebeverlust}

Vollständiger Gewebeverlust mit freiliegenden Knochen, Sehnen oder Muskeln ( $\triangleright$ Abb. 4.8). Beläge oder Schorf können an einigen Teilen des Wundbettes vorhanden sein. Es können Taschenbildungen oder Unterminierungen vorliegen.

Die Tiefe eines Dekubitus der Kategorie IV variiert je nach anatomischer Lokalisation. Der Nasenrücken, das Ohr, das Hinterhaupt und der Knöchel haben kein subkutanes Gewebe und diese Ulzera können oberflächlich sein. Ulzera der Kategorie IV können sich in Muskeln und/oder unterstützenden Strukturen ausbreiten (z. B. Faszie, Sehne oder Gelenkkapsel) und eine Osteomyelitis verursachen. Offenliegende Knochen/Sehnen sind sichtbar oder direkt tastbar. 


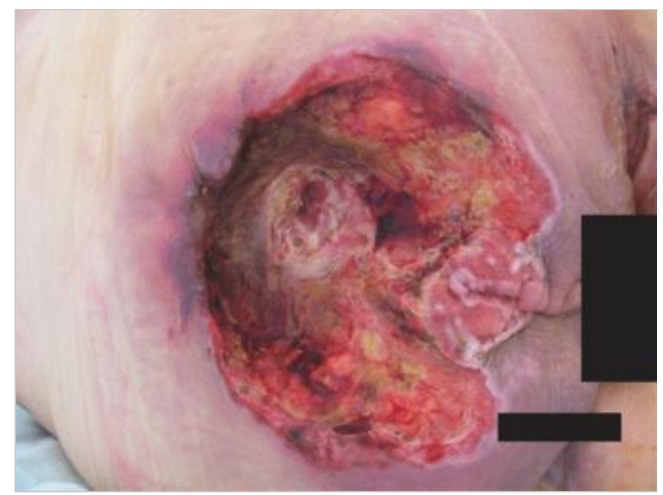

Abb. 4.8 Dekubitus

Stadium IV.

\section{Keiner Kategorie/keinem Stadium zuordenbar: Tiefe unbekannt}

Ein vollständiger Gewebeverlust, bei dem die Basis des Ulkus von Belägen (gelb, hellbraun, grau, grün oder braun) und/oder Schorf im Wundbett bedeckt ist ( $\triangleright$ Abb. 4.9).

Bis genügend Beläge und/oder Schorf entfernt ist, um den Grund der Wunde offenzulegen, kann die wirkliche Tiefe - und daher die Kategorie - nicht festgestellt werden.

\section{Merke}

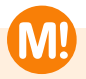

Stabiler Schorf (trocken, festhaftend, intakt ohne Erythem und Flüssigkeit) an den Fersen dient als „natürlicher (biologischer) Schutz des Körpers“ und sollte nicht entfernt werden!

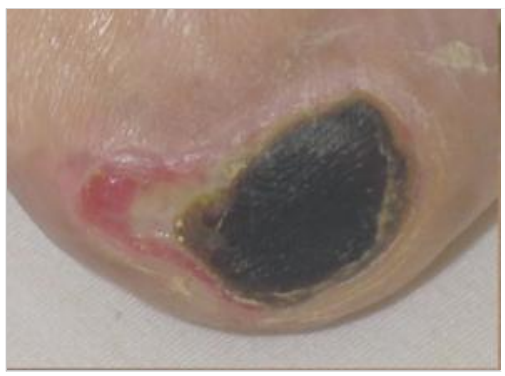

Abb.4.9 Dekubitus mit Schorf, Tiefe unbekannt, keinem Stadium zuzuordnen. 
Abb.4.10 Dekubitus mit vermuteter tiefer Gewebeschädigung, Tiefe unbekannt.

\section{Vermutete tiefe Gewebeschädigung: Tiefe unbekannt}

Livid oder rötlich-brauner, lokalisierter Bereich von verfärbter, intakter Haut oder blutgefüllte Blase aufgrund einer Schädigung des darunterliegenden Weichgewebes durch Druck und/oder Scherkräfte ( $\vee$ Abb. 4.10). Diesem Bereich vorausgehen kann Gewebe, das schmerzhaft, fest, breiig, matschig, im Vergleich zu dem umliegenden Gewebe wärmer oder kälter ist.

Bei der Entstehung kann es zu einer dünnen Blase über einem dunklen Wundbett kommen. Die Wunde kann sich weiter verändern und von einem dünnen Schorf bedeckt sein. Auch unter optimaler Behandlung kann es zu einem rasanten Verlauf unter Freilegung weiterer Gewebeschichten kommen.

\subsection{IAD: Inkontinenz-assoziierte Dermatitis}

\subsubsection{Pathogenese}

Ständiger Kontakt mit Stuhl/Urin schädigt die Hautbarriere und irritiert die Haut. Dadurch werden Entzündungen im Sinne einer irritativ toxischen Kontaktdermatitis ausgelöst, die zunächst als Rötungen, Ekzem und Mazerationen erscheinen. Diese können sich bis zum Vollbild einer IAD ausprägen ( $\triangleright$ Abb. 4.11, $\triangleright$ Abb. 4.12).

Eine IAD ist von einem Dekubitus (s. Kap. 4.1) abzugrenzen; es kann auch beides kombiniert vorliegen.

Risikofaktoren:

- Harninkontinenz und/oder Stuhlinkontinenz

- hohe Entleerungsfrequenz mit flüssigem Stuhl

- ältere Patienten mit Mobilitätseinschränkung und/oder Pflegebedürftigkeit

- sensorische Einschränkungen. 


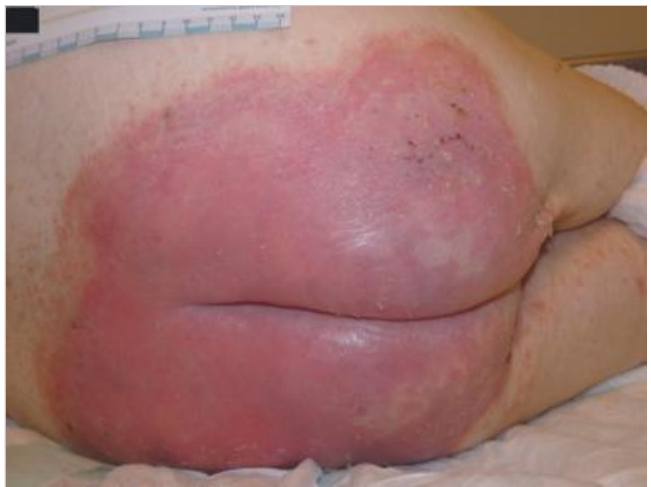

Abb.4.11 IAD Stadium 1.

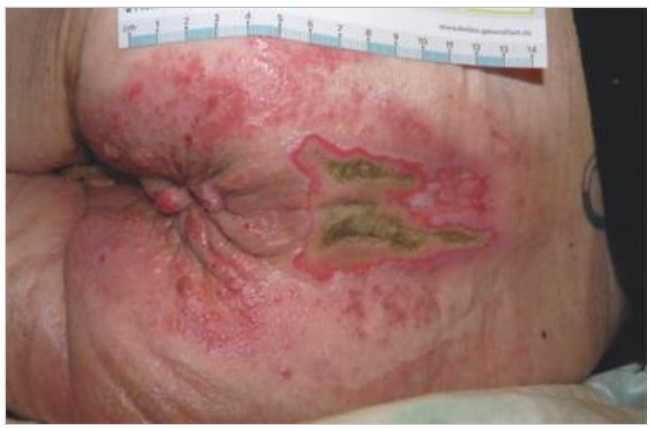

Abb. 4.12 IAD Stadium 2.

\subsubsection{Symptome}

- Verlust der Haut-Barrierefunktion durch Schädigung/Zerstörung der Epidermis

- Hautrötung, Überwärmung, Schwellung, Schuppung, Bildung von Bläschen oder Krusten

- Mazeration der Haut

- nässende Erosionen an der Hautoberfläche

- lokale Blutungen/Erosionen

- Infektionen mit z. B. Candida albicans, Escherichia coli, Streptokokken, Staphylococcus aureus

- Schmerzen

- evtl. Juckreiz

- evtl. Ammoniakgeruch. 


\section{Einteilung:}

- gefährdeter Patient mit Stuhlinkontinenz, Harninkontinenz mit noch intakter Haut

- Kategorie 1 - gerötete aber intakte Haut mit mildem Erythem und Ödem

- Kategorie 2 - gerötet mit Hautschäden zusätzlich Bläschen, Bullae, Hauterosion, Denudation der Haut oder Hautinfektion.

\subsubsection{Unterscheidung IAD vs. Dekubitus}

Die Unterscheidung der beiden Erkrankungen ( $\triangleright$ Tab. 4.1) ist notwendig, damit der Betroffene die richtige Therapie bekommt und die Dokumentation korrekt erfolgt.

Die IAD beginnt „Von außen nach innen“: Zunächst entsteht der Schaden durch die Inkontinenz auf der Oberfläche der Haut. Beim Dekubitus dagegen beginnt die Schädigung innerhalb des Hautgewebes durch Druck oder durch Druck in Kombination mit Scherkräften und setzt sich von „innen nach außen“ fort.

Die IAD braucht Feuchtigkeit, hat keine Nekrosen, ist eher diffus berandet (schlecht differenzierte Ränder) und zeigt manchmal (Cave: nicht immer!) ein wegdrückbares rosafarbenes bis rotes Erythem.

\section{Merke}

Eine IAD liegt bei kontinenten Patienten nicht vor.

\subsubsection{Therapie}

- Inkontinenzbehandlung

- Behandlung reversibler Ursachen der Inkontinenz

- Optimierung der Ernährung, Flüssigkeitszufuhr

- Durchführung der Toilettenhygiene

- Dekubitus-Prävention

- tägliche Hautpflege und ggf. nach Stuhlinkontamination

- Reinigen der irritierenden Substanzen wie Urin und/oder Stuhl mit antibakterieller Waschlotion oder einem Feuchttuch; Cave: keine Seife verwenden!

- Hautschutz mit einem Film (Barriere), der den direkten Kontakt mit Urin / Stuhl verhindert z. B. Cavilon, Cavilon advanced (s. Kap. 5.1), ggf. Stuhldrainagesystem, Fäkalkollektor

- Aufbau der Hautbarriere mit topischem Hautpflegeprodukt 
Tab. 4.1 Unterschied IAD, Dekubitus, ITD

Cave: Kombinationen aus Dekubitus und IAD und ITD können auftreten.

\begin{tabular}{|c|c|c|c|}
\hline & $\begin{array}{l}\text { Dekubitus } \\
\text { (Button-up-Läsion) }\end{array}$ & $\begin{array}{l}\text { IAD } \\
\text { Inkontinenzasso- } \\
\text { ziierte Dermatitis } \\
\text { (Top-down-Läsion) }\end{array}$ & $\begin{array}{l}\text { ITD } \\
\text { Intertriginöse } \\
\text { Dermatitis }\end{array}$ \\
\hline Ursache & $\begin{array}{l}\text { Druck- und/oder } \\
\text { Scherkräfte }\end{array}$ & $\begin{array}{l}\text { Feuchtigkeit } \\
\text { (Harninkontinenz, } \\
\text { Diarrhoe) }\end{array}$ & $\begin{array}{l}\text { Reibung, gestaute } \\
\text { Wärme, Feuchtig- } \\
\text { keit, Diaphorese }\end{array}$ \\
\hline Lokalisation & $\begin{array}{l}\text { über knochigen } \\
\text { prominenten Stel- } \\
\text { len oder unter me- } \\
\text { dizinischen Geräten }\end{array}$ & $\begin{array}{l}\text { Perineum, perianale } \\
\text { Umgebung, innere } \\
\text { Schenkel, Gesäß, } \\
\text { kann auch über } \\
\text { Knochenvorsprün- } \\
\text { gen liegen (Druck- } \\
\text { und Scherkräfte } \\
\text { ausschließen!) }\end{array}$ & $\begin{array}{l}\text { Gesäßfalte, Leisten- } \\
\text { beuge, Bauchfalte, } \\
\text { submammär (ge- } \\
\text { genüberliegende } \\
\text { Hautoberfläche) }\end{array}$ \\
\hline Ausprägung & lokal begrenzt & $\begin{array}{l}\text { diffuse, oberflächli- } \\
\text { che Hautverände- } \\
\text { rung }\end{array}$ & $\begin{array}{l}\text { oberflächliche leich- } \\
\text { te bzw. starke Rö- } \\
\text { tung, spiegelbildlich } \\
\text { auf beiden Seiten } \\
\text { der Hautfalte }\end{array}$ \\
\hline Tiefe & $\begin{array}{l}\text { bis Knochen, Mus- } \\
\text { keln möglich }\end{array}$ & $\begin{array}{l}\text { normalerweise } \\
\text { oberflächliches Ge- } \\
\text { webe, teilweise feh- } \\
\text { lende Hautschicht }\end{array}$ & $\begin{array}{l}\text { normalerweise } \\
\text { oberflächlich zu } \\
\text { Beginn, mazerativ } \\
\text { bis erosiv }\end{array}$ \\
\hline Hautrötung & $\begin{array}{l}\text { nicht wegdrück- } \\
\text { bares Erythem bei } \\
\text { Kategorie } 1\end{array}$ & $\begin{array}{l}\text { wegdrückbares } \\
\text { oder nicht weg- } \\
\text { drückbares Erythem }\end{array}$ & $\begin{array}{l}\text { wegdrückbares } \\
\text { Erythem }\end{array}$ \\
\hline Wundränder & $\begin{array}{l}\text { typischerweise } \\
\text { rund; wenn Scher- } \\
\text { kräfte involviert } \\
\text { sind, oval oder } \\
\text { länglich, klare Rän- } \\
\text { der }\end{array}$ & $\begin{array}{l}\text { diffuse und unglei- } \\
\text { che Ränder }\end{array}$ & $\begin{array}{l}\text { lineare Verletzung } \\
\text { der Haut, Satelliten- } \\
\text { papeln }\end{array}$ \\
\hline Wundfläche & $\begin{array}{l}\text { Unterminierung } \\
\text { und Taschenbil- } \\
\text { dung, Nekrosen } \\
\text { möglich }\end{array}$ & $\begin{array}{l}\text { keine Nekrosen, } \\
\text { Wundumgebung } \\
\text { mazeriert }\end{array}$ & $\begin{array}{l}\text { keine Nekrosen, } \\
\text { Hautumgebung } \\
\text { mazeriert }\end{array}$ \\
\hline
\end{tabular}




\subsection{Verbrennung}

\subsubsection{Definition und Pathogenese}

Unter Verbrennung versteht man im Allgemeinen eine thermische Verletzung durch Temperaturen, welche die Regulationsfähigkeit der Haut überfordern und zu Gewebeschädigungen führen. Dies kann durch heiße Flüssigkeiten (Verbrühung), Dämpfe oder Gase, heiße Stoffe oder Kontaktflächen, Flammeneinwirkung und Explosionen, starke Sonneneinstrahlung, elektrischen Strom oder Reibung entstehen.

Auch chemische Noxen (Säuren oder Laugen) können ähnliche Schädigungen verursachen. Je nach Höhe der Temperatur und Zeitdauer der Hitzeeinwirkung entstehen Gewebeschäden primär an Haut und Schleimhäuten oder an tieferen Gewebsstrukturen mit Nekrose und Gewebsuntergang. Verbrennungsverletzungen, die ein bestimmtes Maß überschreiten, können in Abhängigkeit vom Ausmaß der unmittelbaren Schädigung zusätzlich zu Kreislaufschock und entzündlichen Allgemeinreaktionen des Körpers im Rahmen eines SIRS oder einer Sepsis führen, die im schlimmsten Fall mit Multiorgandysfunktion oder -versagen verbunden sind. Die Gesamtheit dieser systemischen Störungen bezeichnet man als Verbrennungskrankheit.

\subsubsection{Einteilung}

Der Schweregrad einer Brandverletzung bemisst sich nach Ausmaß und Tiefe der verbrannten Oberfläche ( $\triangleright$ Tab. 4.2, $\triangleright$ Abb. 4.13). Zusätzlich ist die Art der

Tab. 4.2 Einteilung von Verbrennungen.

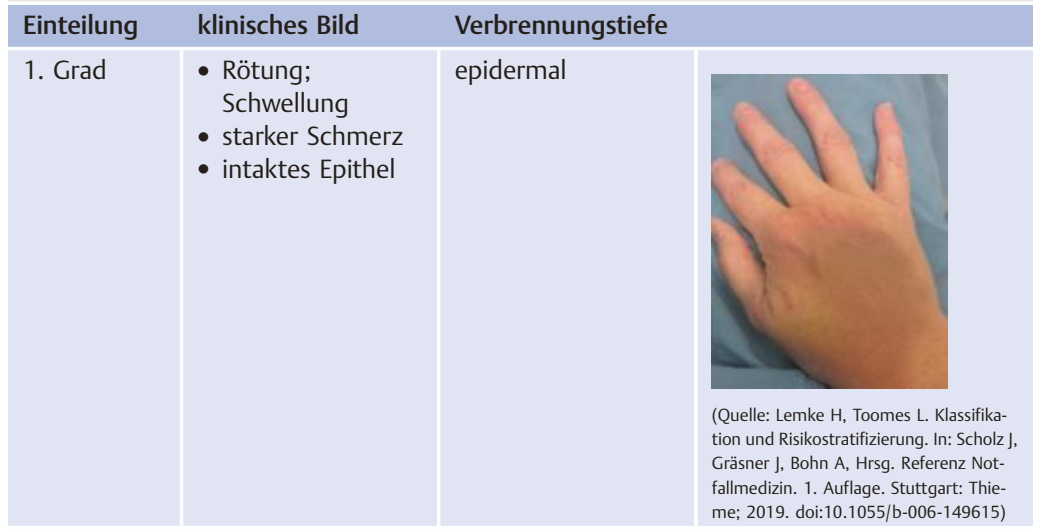


Tab. 4.2 Fortsetzung.

\begin{tabular}{|c|c|c|c|}
\hline Einteilung & klinisches Bild & Verbrennungstiefe & \\
\hline 2. Grad a & $\begin{array}{l}\text { - Blasenbildung } \\
\text { - feuchter, hyper- } \\
\text { ämischer Unter- } \\
\text { grund } \\
\text { - stark schmerzhaft }\end{array}$ & $\begin{array}{l}\text { Schädigung der } \\
\text { Epidermis und } \\
\text { oberflächlicher An- } \\
\text { teile der Dermis mit } \\
\text { Sequestrierung }\end{array}$ & $\begin{array}{l}\text { (Quelle: Paetz B. Tiefenausdehnung. } \\
\text { In: Paetz B, Hrsg. Chirurgie für Pflege- } \\
\text { berufe. . 23,. überarbeitete Auflage. } \\
\text { Stuttgart: Thieme; 2017. doi:10.1055/ } \\
\text { b-005-143670) }\end{array}$ \\
\hline 2. Grad b & $\begin{array}{l}\text { - } \text { fetzenförmige } \\
\text { Epidermolyse } \\
\text { - Blasenbildung } \\
\text { - weißlicher, feuch- } \\
\text { ter Wundgrund } \\
\text { - gestörte Rekapil- } \\
\text { larisierung } \\
\text { - mäßiger Schmerz }\end{array}$ & $\begin{array}{l}\text { weitgehende Schä- } \\
\text { digung der Dermis } \\
\text { unter teilweiser } \\
\text { Zerstörung der } \\
\text { Haarfollikel und } \\
\text { Drüsenanhängsel }\end{array}$ & 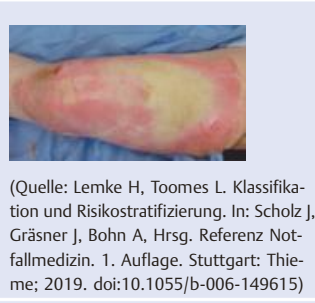 \\
\hline 3. Grad & $\begin{array}{l}\text { - Gewebe nach } \\
\text { Reinigung weiß } \\
\text { - keine Schmerzen }\end{array}$ & $\begin{array}{l}\text { vollständige Zerstö- } \\
\text { rung von Epidermis } \\
\text { und Dermis }\end{array}$ & $\begin{array}{l}\text { (Quelle: Lemke H. Toomes L. Klassifika- } \\
\text { tion nund Risikstratifizierung. In: ccholz, } \\
\text { Gränner J, Bohn A, Hrsg. Referenz Not- } \\
\text { fallmedizin. 1. Auflage. Stuttgart: Thie- } \\
\text { me; 2019. doi:10.1055/b-006-149615) }\end{array}$ \\
\hline 4. Grad & $\begin{array}{l}\text { Verkohlung, Lyse } \\
\text { (bei chemischer } \\
\text { Schädigung) }\end{array}$ & $\begin{array}{l}\text { Zerstörung weit- } \\
\text { gehender Schichten } \\
\text { mit Unterhautfett- } \\
\text { gewebe, eventuell } \\
\text { Muskeln, Sehnen, } \\
\text { Knochen und Ge- } \\
\text { lenken }\end{array}$ & $\begin{array}{l}\text { (Quelle: Lemke H, Toomes L. Klassifika- } \\
\text { tion und Risikostratifizierung. II: Ccholl J, } \\
\text { Gräsner J, Bohn A, Hrsg. Referenz Not- } \\
\text { fallmedizin. 1. Auflage. Stuttgart: Thie- } \\
\text { me; 2019. doi:10.1055/b-006-149615) }\end{array}$ \\
\hline
\end{tabular}




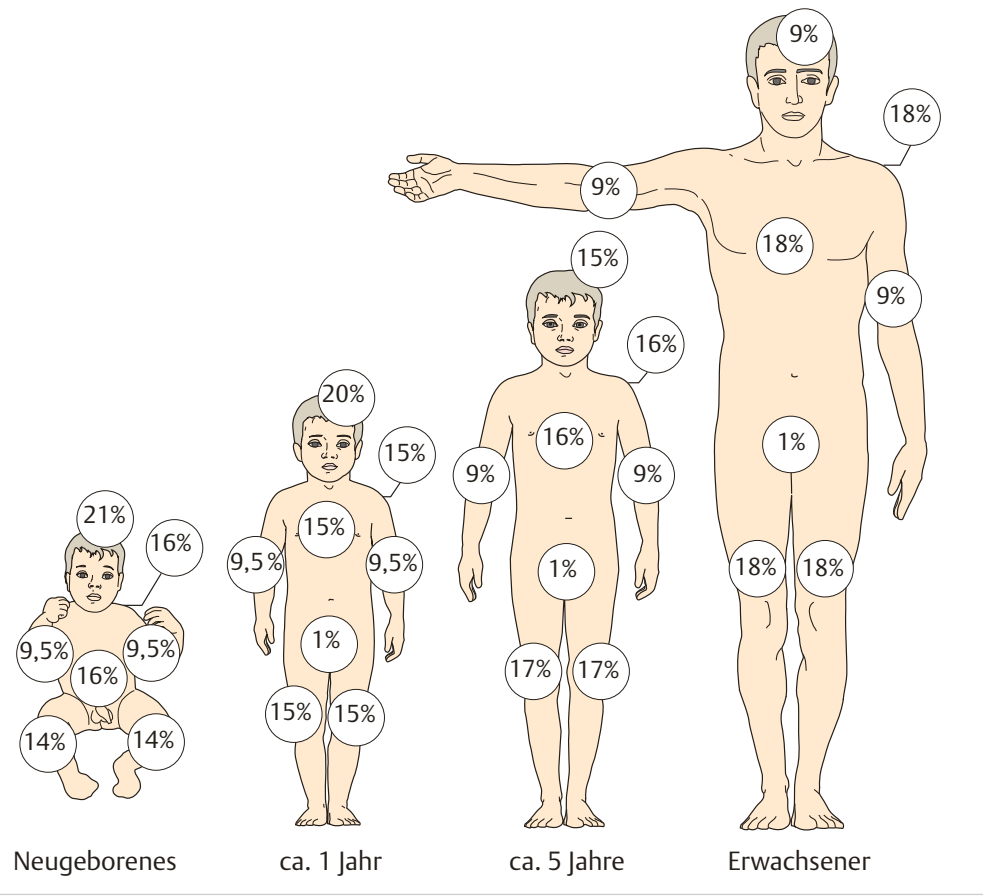

Abb.4.13 Berechnung des Ausmaßes einer Brandverletzung (in Prozent der Körperoberfläche). (Quelle: Kempf A, Mehlig U. Epidemiologie. In: Kretz F, Becke K, Eberius C, Hrsg. Anästhesie bei Kindern. 4., vollständig überarbeitete Auflage. Stuttgart: Thieme; 2019. doi:10.1055/b-007-170420)

Noxe (Verbrennung/Verbrühung/Säure) relevant. Eine sekundäre Ausdehnung ist nach 24 Stunden erneut zu beurteilen.

Begleitende Verletzungen, wie ein Inhalationstrauma oder beispielsweise Frakturen im Rahmen eines Explosionstraumas, verstärken die Verletzung zusätzlich. 


\subsubsection{Therapie}

Je nach Verbrennungsausmaß, Lokalisation und ggf. Zusatzverletzungen bemisst sich das weitere therapeutische Vorgehen.

\section{Erstmaßnahmen}

- Überprüfung und Sicherung der Vitalfunktionen

- Abklärung hinsichtlich des Ausmaßes und der Tiefe

- Behandlung von Begleitverletzungen

- Analgosedierung

- Kühlung:

○ kleinere Verbrennungen: lauwarmes Wasser vor Ort für 10-20 Minuten

- Verzicht auf Kühlung bei großflächigen Verletzungen > 15\% der Körperoberfläche (KOF), Kleinkinder, Säuglinge, Neugeborene, intubierte und beatmete Patienten

- Cave: Erhalt der Körperkerntemperatur; Hypothermie geht mit einer signifikant erhöhten Letalität einher

- Abdecken der Wunde steril

- Adäquate Volumentherapie, auch als Infusionstherapie mit isotonen, kristalloiden Lösungen. Menge von $10 \mathrm{ml} / \mathrm{kg}$ Körpergewicht/h nicht überschreiten.

\section{Cave}

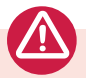

An eine CO Vergiftung denken!

\section{Behandlung in der Klinik}

Die Behandlung richtet sich nach der Ausdehnung und Tiefe der Verletzungen, der Lokalisation und den Begleitverletzungen. Die Indikation zur Verlegung in ein Zentrum erfolgt entsprechend der Leitlinie der Deutschen Gesellschaft für Verbrennungsmedizin.

Ein sicherer venöser Zugang sollte für die Möglichkeit einer Volumentherapie und einer ausreichenden Schmerztherapie angelegt werden. 


\section{Flüssigkeitssubstitution}

Bei einer Verbrennung besteht ein erheblicher zusätzlicher Flüssigkeitsbedarf (Verbrennungskrankheit, Volumenmangel). Mit der Parkland-Formel (zur Berechnung der benötigten Flüssigkeitsmenge) oder der modifizierten ParklandFormel für Kinder kann der zusätzliche Flüssigkeitsbedarf berechnet werden.

\section{Lokale Therapie}

Alle Verbandwechsel sind unter aseptischen Bedingungen und möglichst schmerzarm durchzuführen.

Grad 1: Die Behandlung erfolgt konservativ mit z. B. rückfettender Hautpflege (Panthenol) und/oder Anwendung von Wunddistanzgittern. Sorgfältige Reinigung des Areals mit Wasser, Seife oder wässrigem Antiseptikum. Eine lokale Kühlung durch feucht-kühle Umschläge und eine orale Basisanalgesie sollte in der ambulanten Therapieempfehlung vorgesehen werden.

Grad 2a: Die Behandlung ist konservativ.

Die Wundreinigung mit Débridement erfolgt unter aseptischen Bedingungen in Analgosedierung oder Narkose. Für die anschließende Versorgung werden Okklusivverbände mit inaktiven, aktiven oder biologischen Verbandsystemen verwendet. Individuell können, ggf. auch zeitlich verzögert, temporäre synthetische oder biologische Hautersatzmaterialien Anwendung finden.

Empfehlung zur Behandlung:

- Wundversorgung in der Notaufnahme oder im OP

- Wundreinigung unter aseptischen Bedingungen mit Lavasorb

- Rasur des angrenzenden Areals

- kleinere Areale: Verband mit Wunddistanzgittern (fetthaltig, z. B. Jelonet) und Prontosan Wound Gel X, darüber trockene Kompressen und Fixomull oder halbelastisches Verbandsmaterial

- größere Areale: Aufbringen von Suprathel oder Biobrane, breit überlappendes Wunddistanzgitter (fetthaltig, z. B. Jelonet) sowie trockene Kompressen und Fixomull oder halbelastisches Verbandsmaterial; auf eine straffe Fixierung der Hautersatzmaterialien muss beim Aufbringen geachtet werden. 
Grad 2b: Bei eindeutiger Tiefenzuordnung und Demarkierung wird zeitnah eine operative tangentiale Exzision bis in punktuell blutende Dermisanteile durchgeführt. In Abhängigkeit von Lokalisation, Größe und Zeitpunkt werden diese Bereiche mit aktiven oder biologischen Wundmanagementsystemen, synthetischem oder biologischem epidermalem Hautersatzmaterial oder autologer Spalthaut abgedeckt und versorgt. Bei fraglicher Zuordnung (2a/2b) kann die eindeutige Demarkierung je nach Lokalisation und Ausdehnung sowie Ursache der thermischen Schädigung bis zu 2 Wochen abgewartet werden. Bis dahin wird konservativ mit antiseptischen Verbänden oder temporärem epidermalem Hautersatzmaterial behandelt.

Grad 3: Nach eindeutiger Abschätzung der Tiefe wird zeitnah oder sequenziell eine tangentiale oder epifasziale Nekrektomie durchgeführt. Anschließend erfolgt die Defektdeckung durch Hauttransplantation oder andere geeignete plastische Verfahren. Gegebenenfalls erfolgt bei großen Defekten eine temporäre Deckung mit biosynthetischen Folien, mit allogener Spalthaut oder in Form der Vakuumversiegelung. Der Einsatz von Dermisersatzmaterial (MatriDerm, Integra, Hyalomatrix) sollte erwogen werden, ggf. auch in Kombination mit Keratinozyten (Regenercell). Diese speziellen Behandlungsstrategien sollten spezialisierten Zentren vorbehalten sein.

Grad 4: Bei Verbrennungen des Grades 4 muss eine Amputation betroffener Extremitäten oder Anteile kritisch geprüft werden. Ansonsten entspricht das Prozedere dem Vorgehen bei Verbrennungen Grad 3.

\subsubsection{Verbrennungsprodukte}

\section{Produkte}

\section{Gelistet in den Helios-Kliniken}

- Prontosan Wound Gel X (BBR) Tube $50 \mathrm{~g}$

- Lavanid Wundgel V + (SER) Tube $40 \mathrm{~g}$

- Lavasorb Wundspüllösung (FRE) $250 \mathrm{ml}$

- Hautersatzprodukte, siehe Kap. 2.9.7), z. B.

- Suprathel (POL) $5 \times 5 \mathrm{~cm}, 9 \times 10 \mathrm{~cm}, 10 \times 18 \mathrm{~cm}$

- BIOBRANE (S\&N) $13 \times 13 \mathrm{~cm}$, Handschuhe Päd, Handschuh M ( $\triangleright$ Abb. 2.97) 


\section{Prontosan Wound Gel X}

Prontosan Wound Gel X enthält Polyhexanid (Polyhexanidanteil 0,1\%) zur Konservierung gegen Keimwachstum und Undecylenamidopropyl-Betain als oberflächenaktive Substanz, ein besonders hochwertiges Tensid mit ausgezeichneter Reinigungswirkung und guter Hautverträglichkeit. Polyhexanid hemmt effektiv das Keimwachstum und dient der Konservierung nach Anbruch des Gebindes. Die besonders wirksame Kombination dieser beiden Inhaltsstoffe löst den Biofilm und ermöglicht eine ausgezeichnete Wundreinigung, die zu einer deutlichen Verkürzung der Wundheilung führt. Das Gel kann bis zu 8 Wochen nach Anbruch beim selben Patienten angewandt werden, solange es keinen direkten Wundkontakt zur Tube gibt. Durch Lagerung der Prontosan Wound Gel X Tuben im Kühlschrank kann man bei der Applikation einen kühlenden Effekt erzielen. Dies ist aber nicht zwingend notwendig.

Prontosan Wound Gel X kann unmittelbar auf oberflächliche Wunden, aber auch in Wundkavitäten appliziert werden.

Anwendung zur Reinigung, Befeuchtung und Dekontamination von:

- akuten infizierten und nicht infizierten Wunden: traumatische Wunden (wie z. B. Risswunden, Schürfwunden oder Stichwunden - falls ein Nahtverschluss indiziert ist, sollte Prontosan Wound Gel X erst nach dem chirurgischen Eingriff verwendet werden)

- chronischen infizierten und nicht infizierten Wunden (inkl. komplexen, therapieresistenten Wunden, Wundtaschen, schwer zugänglichen Wunden)

- postoperativen Wunden

- durch Hitze, chemische Substanzen und nach Strahlentherapie auftretenden Verbrennungen (1. bis 4. Grades)

- Erfrierungen.

\section{Lavanid Wundgel V +}

Lavanid Wundgel V+ ist ein steriles konserviertes Hydrogel mit hoher Viskosität mit sehr guter Gewebeverträglichkeit:

- direktes Auftragen auf die Wunde zum Befeuchten und Reinigen

- zur konservierenden Befeuchtung von Verbänden und Wundauflagen

- Polyhexanidanteil 0,04\%.

Das Gel kann bis zu 4 Wochen nach Anbruch beim selben Patienten angewandt werden, solange es keinen direkten Wundkontakt zur Tube gibt. 


\section{Lavasorb}

Lavasorb ist eine gebrauchsfertige Wundspüllösung zur lokalen Anwendung. Es enthält Polyhexanid (PHMB) und Macrogol (Polyethylenglycol 4000) in wässriger Lösung (Ringer-Lösung). Es besteht eine Zulassung zur Wundspülung und Applikation in Wundkavitäten.

\section{Suprathel und Biobrane}

Suprathel ( $\triangleright$ Abb. 2.102) ist ein alloplastischer resorbierbarer Hautersatz aus einer Polylactatmembran (Polymilchsäure). Nach Aufbringung wird die Membran transparent und ermöglicht so die Beobachtung des Heilungsfortschritts.

Suprathel wird einmalig aufgebracht. Eine operative Entfernung ist nicht erforderlich, da Suprathel innerhalb von 4-6 Wochen degradiert wird. Suprathel löst sich an wiederhergestellten Hautarealen ab (Anwendung s. D Abb. 4.14).

Biobrane ist ein temporärer alloplastischer, nicht resorbierbarer Hautersatz aus einer semipermeablen Silikonmembran mit 3-D Nylongewebe besetzt mit porcinem Kollagen und Polygluconat ( $\triangleright$ Abb. 2.98, $\triangleright$ Abb. 2.99). Der Hautersatz wird mittels Verbindung von Blut und Serum mit dem Gewebe hergestellt. Biobrane hat Öffnungen zum Austritt von Exsudat und kann auf der Wunde verbleiben, bis die Oberfläche neu epithelisiert ist.

Beide Produkte sind sehr gut auch an Körperteile wie Hände (Biobrane Glove, > Abb. 2.100), Füße und Gesicht anpassbar.

Die Gliedmaßen können darunter ohne Verlust der Wundabdeckung mobilisiert werden.

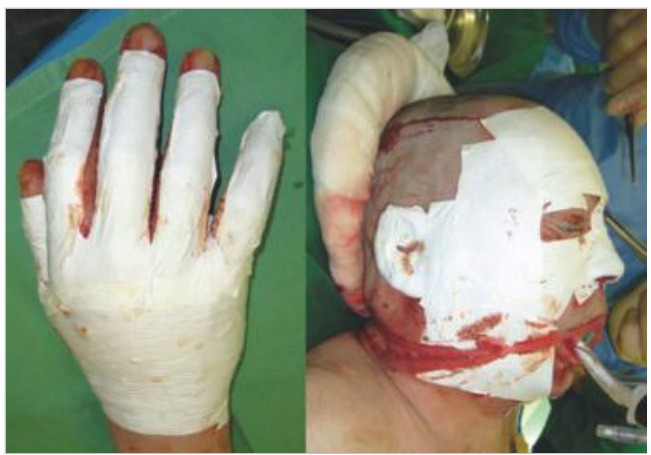

Abb. 4.14 Suprathel: Anwendung. (Quelle: Mit freundlicher Genehmigung von PolyMedics Innovations $\mathrm{GmbH}$ ) 


\section{Indikation}

Thermische Verletzungen, Stromverletzungen, Verätzungen durch Säuren und Laugen Grad 2a-2b, Spalthautentnahmestellen; Suprathel auch bei Wunden mit partiellen Anteilen von Grad 3.

\section{Merke}

Aufgrund der temporären Abdeckung der Wundflächen sind Verbandwechsel des Sekundärverbandes schmerzarm bis schmerzfrei möglich.

Die Anwendung von Biobrane-Handschuhen erleichtert die Versorgung komplexer Verletzungen an der Hand.

\section{Kontraindikationen}

- eröffnete Blasen $>1 \mathrm{~h}$ ohne sterile Abdeckung

- thermische Verletzungen, Stromverletzungen, Verätzungen durch Säuren und Laugen Grad 1 und (3-)4

- ältere Verletzungen mit klinisch bereits infizierten Wunden.

\section{Anwendungsweise}

Die Brandblasen sollten unter sterilen Bedingungen vollständig abgetragen werden. Das verletzte Areal sollte rasiert werden. Unter sterilen Bedingungen kann dann der Verbandsstoff aufgebracht werden ( $\triangleright$ Abb. 4.15).

- Suprathel: entsprechend der Form und Größe der Wunde wird der Verband geformt oder zugeschnitten und locker, nicht überlappend aufgelegt.

- Biobrane: Biobrane muss mit der korrekten Seite zur Wunde liegend aufgebracht werden. Fixierung mittels Kompressionsverband, ggf. Naht oder Klammermaterial. Die Entfernung des Klammer-, bzw. Nahtmaterials kann nach 4 Tagen, nach Erreichen einer festen Verbindung zwischen Biobrane und dem Wundgrund, erfolgen. Die Abtragung von Biobrane selbst erfolgt tangential von der Seite der Verletzung unter steter Entfernung der sich lösenden Areale. 


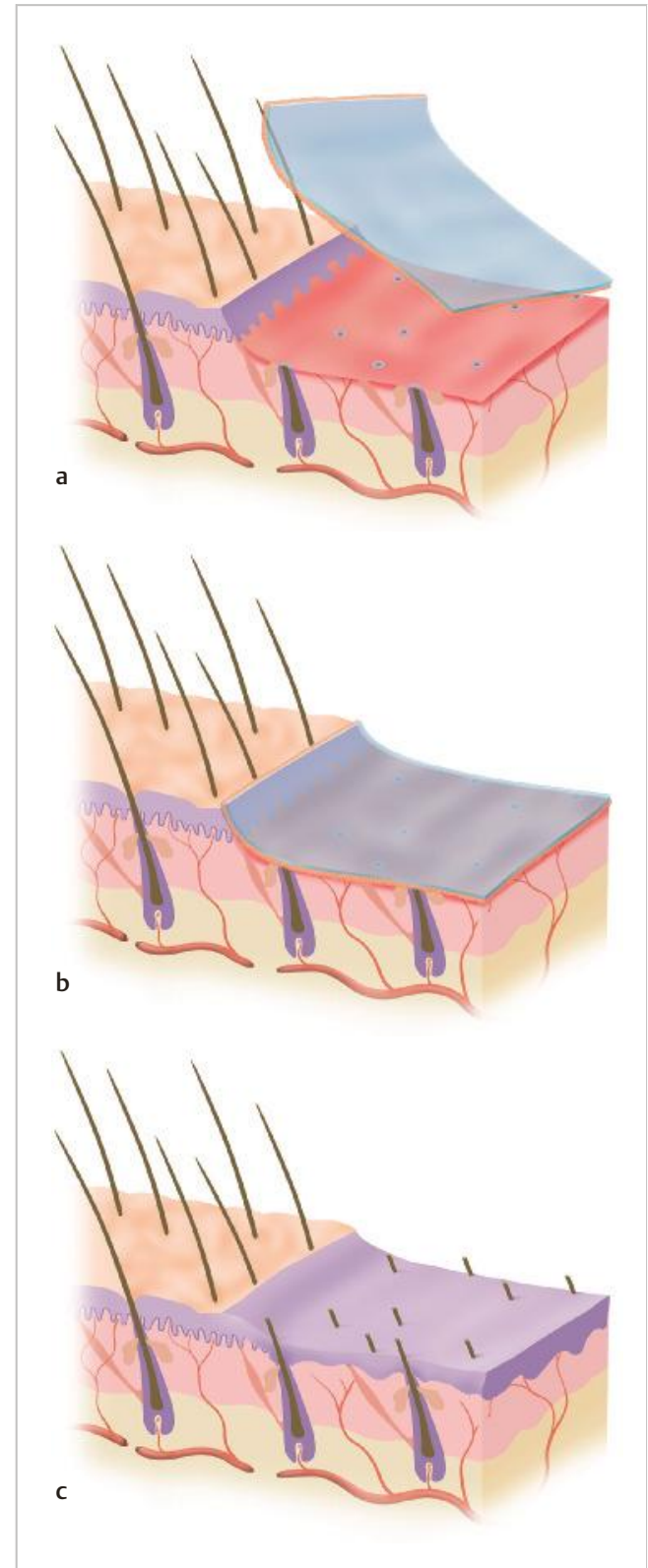

\section{Abb.4.15 Anwendungs-} weise von Biobrane.

a Aufbringen von Biobrane nach oberflächlichem Débridement bei erhaltenen Haarfollikeln.

b Anmodellierung mit flächigem Kontakt.

c Regeneration der Epidermis aus den Haarfollikeln und den seitlichen Rändern. 


\section{Merke}

Bei beiden Produkten sollte zum Schutz des Wundareals vor mechanischer Alteration eine einlagige Abdeckung mit einem fetthaltigen Wunddistanzgitter erfolgen. Darüber kann ein Mullverband gegen mechanische Alteration oder Verschmutzung schützen. Dieser Verband kann schmerzfrei gewechselt werden.

Beide Produkte und die fettige Gaze können bis zur Abheilung der Wunde belassen werden.

Bei Kindern ist in der Regel die Anlage in Sedierung sinnvoll.

Verbandwechsel:

- normalerweise genügt ein Verbandwechsel des Sekundärverbandes alle 2-3 Tage.

- im Rahmen jedes Verbandwechsels können die lockeren Seitenränder der Produkte abgetragen werde

- nach spätestens 14 Tagen kann die restliche Wundauflage entfernt werden

- verbliebene Wunden bedürfen in der Regel einer chirurgischen Sanierung.

\section{Merke}

Im Rahmen des Verbandwechsels sollte ein fetthaltiges Wunddistanzgitter mit dem darunter befindlichen Hautersatz auf der Wunde verbleiben. Ein vorsichtiges Ablösen des Überverbandes ist zwingend notwendig, um einen Verlust des Hautersatzes zu vermeiden. Im Rahmen des Verbandwechsels sollte keine Feuchtigkeit auf den Verband aufgebracht werden.

\section{Cave}

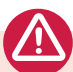

Bei Anzeichen einer Wundinfektion ist der Hautersatz umgehend zu entfernen. 


\subsection{Offenes Abdomen}

Das „offene Abdomen“ hat sich in der Chirurgie als Behandlungsstrategie bei Polytraumapatienten, dem abdominellem Kompartmentsyndrom und schweren intraabdominellen Infektionen etabliert. Es kann auf verschiedene Arten verschlossen, bzw. die Wundränder angenähert werden. Ziel ist es, die Faszienränder einander anzunähern, um sekundär auch nach mehreren Eingriffen einen kompletten Bauchdeckenverschluss zu erreichen.

\subsubsection{Konventionelle Versorgung des offenen Abdomens}

Hierbei erfolgt die Einnaht eines oder mehrerer resorbierbarer oder nicht resorbierbarer Netze in die Faszienränder ( $\vee$ Abb. 4.16). Das Abdomen wird mit einer Folie verschlossen und über Klebebeutel eine Drainage erreicht. Die massive Exsudatmenge stellt dabei ein großes Problem dar. Drainagen oder Klebebeutel versagen hier oft, der Verband muss sehr oft gewechselt werden. Es besteht die Gefahr der Mazeration der Umgebungshaut. Die unmittelbare Behandlung erfolgt mit Cavilon, in schweren Fällen mit Cavilon Advanced (s. Kap. 5.1.3).

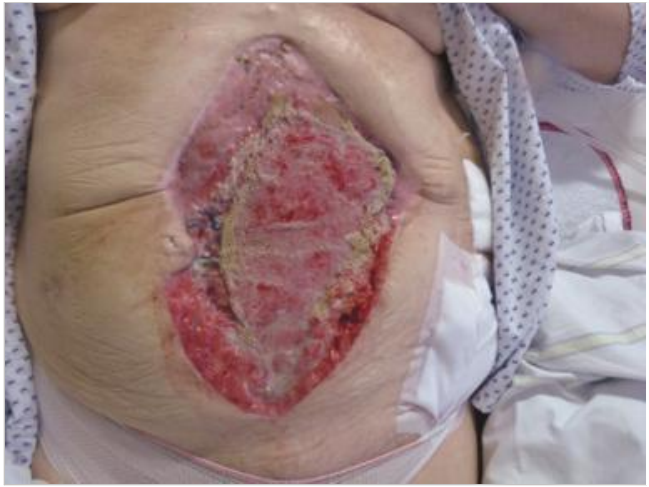

Abb. 4.16 Bauchdecke mit Wundheilungsstörung nach offenem Abdomen. Versorgung mit Vicrylnetz. 


\subsubsection{Intraabdominale Negative Pressure Wound Therapy (NPWT)}

Bei diesem Verfahren wird eine erste Schicht aus einer perforierten PE-Folie intraabdominell eingebracht ( $\triangleright$ Abb. 4.17). Diese dient dem Schutz vor viszeroabdominellen Wandadhäsionen. Je nach Hersteller befindet sich der Schwamm in oder auf der Folie. Bei Anpassung des Schwamms sollte dieser nicht bis zu den Wundrändern reichen. Es empfiehlt sich ein ca. 0,5-1 cm großer Abstand zum Wundrand.

Nach Einbringen des Schwamms wird die Wunde mit einer selbstklebenden Folie verschlossen und ein kontinuierlicher Sog mit 60-120 mmHg angelegt.

Wechsel des Systems: alle 48-72 Stunden mit schrittweisem, spannungsfreiem Bauchdeckenverschluss.

\section{Cave}

- bei Infektion sofortiger Wechsel des Systems

- kein direkter Kontakt des Darms mit dem Schwamm $\rightarrow$ Fistelgefahr!

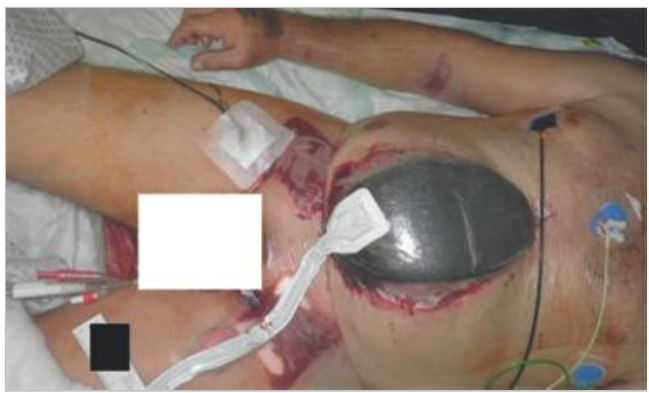

Abb.4.17 Abdominelle NPWT. 


\subsubsection{Kombination der Verfahren Netzeinbau mit Negative Pressure Wound Therapy (NPWT)}

Hierbei wird das Abdomen mittels eines Netzes soweit wie möglich spannungsfrei verschlossen bzw. die Ränder angenähert ( $\triangleright$ Abb. 4.18). Auf das Netz wird ein konventionelles NPWT-System aufgebracht. Der Vorteil gegenüber der konventionellen Behandlung ist das bessere Exsudat-Management. Jedoch sollte hier zum Schutz vor einer Fistelbildung eine Silikonauflage wie z.B. Mepitel One zwischen Schwamm und Netz gelegt werden. Ansonsten erfolgt die Anlage der NPWT-Therapie wie gewohnt.

\subsubsection{Komplikation: intestinale Fistel}

Eine schwere Komplikation bei der Behandlung des offenen Abdomens ist die Ausbildung einer intestinalen Fistel. Während eine enterokutane Fistel mittels eines AP Beutels versorgt werden kann, ergeben sich große Probleme beim Versorgen des Fistelinhaltes und gleichzeitigem Schutz der umgebenden Wunde. Versuche, die Fistel operativ zu sanieren, sind meistens nicht erfolgreich.

Eine Standardversorgung hat sich nicht etabliert, je nach Situation kommen unterschiedliche Verfahrenskombinationen zur Anwendung. Die Restwunde heilt schneller, wenn die Fistel und das Sekret davon separiert werden.

Es gibt hierfür folgende Hilfsmöglichkeiten:

- NPWT und Fisteladapter

- Wunddrainagebeutel.

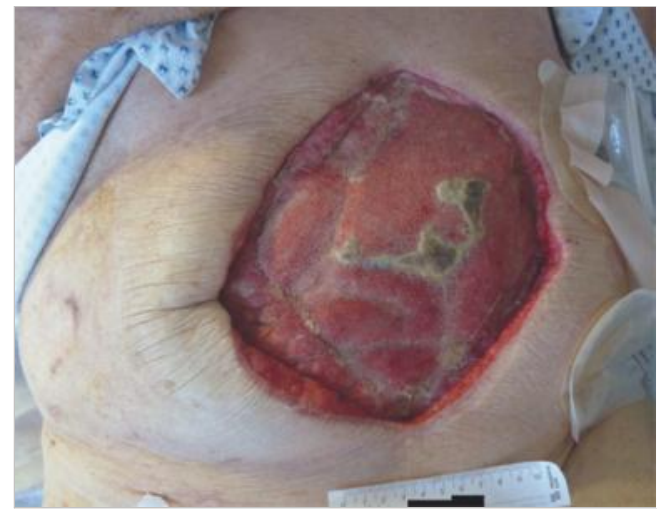

Abb. 4.18 Netzeinlage und Förderung der Granulation mittels NPWT. 


\section{Versorgung mit NPWT und Fisteladapter}

Bei Versorgung mit NPWT ohne Adapter wirkt der Sog direkt auf die Fistelregion, dadurch vergrößert sich diese durch Eventration der Darmmukosa. Das stuhlige Sekret kann zur Verstopfung des Schwamms führen, daraus resultiert dann eine insuffiziente Saugleistung. Stuhlgang unterhalb des Schwamms führt häufig zur Kontamination, Infektion, Sepsis und Wundheilungsstörung.

Einsetzbar sind Fisteladapter in Kombination mit der NPWT-Therapie. Somit wird die Wunde mit Unterdruck therapiert und das Sekret sauber von der Wunde getrennt und abgeleitet.

\section{Beschreibung}

Der Fisteladapter ist ein Zylinder aus einem thermoplastischen Elastomer, welches flexibel ist, um die mechanische Irritation des Darmes zu verringern.

Es gibt verschiedene Durchmesser und Höhen. An der Basis besteht ein breiterer Rand als am oberen Ende.

\section{Anwendung}

Reinigung der Wundfläche. Bestimmung des Durchmessers der Fistel und Aufbringen einer Lage einer nicht verklebenden, perforierten Silikonauflage, z.B. Mepitel One entsprechend der Wundgröße, mit Aussparung der Fistel. Eine Überlappung zwischen Silikonauflage und Fisteladapter ist notwendig, damit der Polyurethan-Schwamm keinen direkten Kontakt zum Darmpaket hat.

Einsetzen des Fisteladapters in eine entsprechend zugeschnittene Öffnung im PU-Schwamm und Aufbringen des Schwamms.

Die Öffnung des Adapters und die Öffnung in der Mepitelauflage decken sich. Anschließend erfolgt das Überkleben der Wunde mit Polyurethanfolie und Anbringen des Sogkonnektors. Die Folie wird über dem Fisteladapter ausgeschnitten und ein zweiteiliges Stoma-Set aufgeklebt. Über den Sogkonnektor (Soft port) wird ein Sog von $60-120 \mathrm{mmHg}$ in der Wunde angelegt. Der Unterdruck hält den Fisteladapter sicher in der gewählten Position ( $\triangleright$ Abb. 4.19). 


\subsection{Offenes Abdomen}
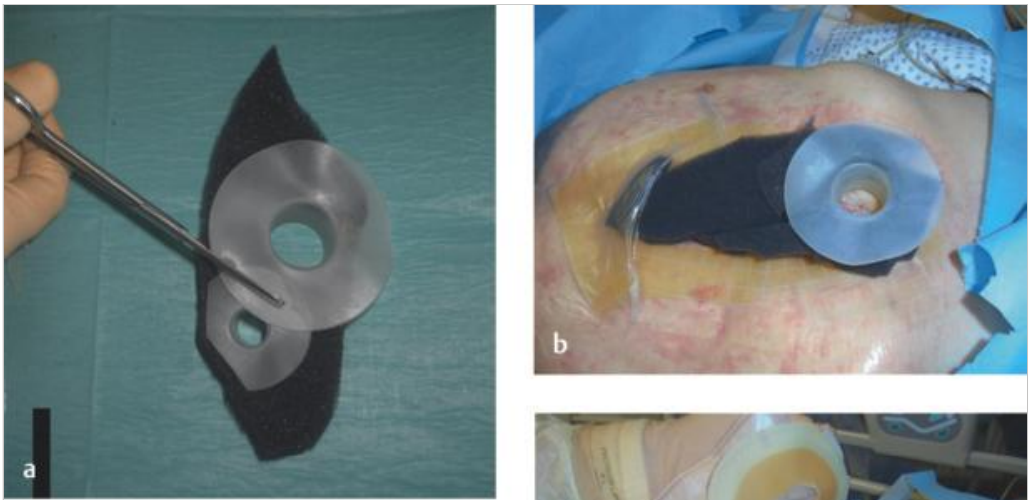

(
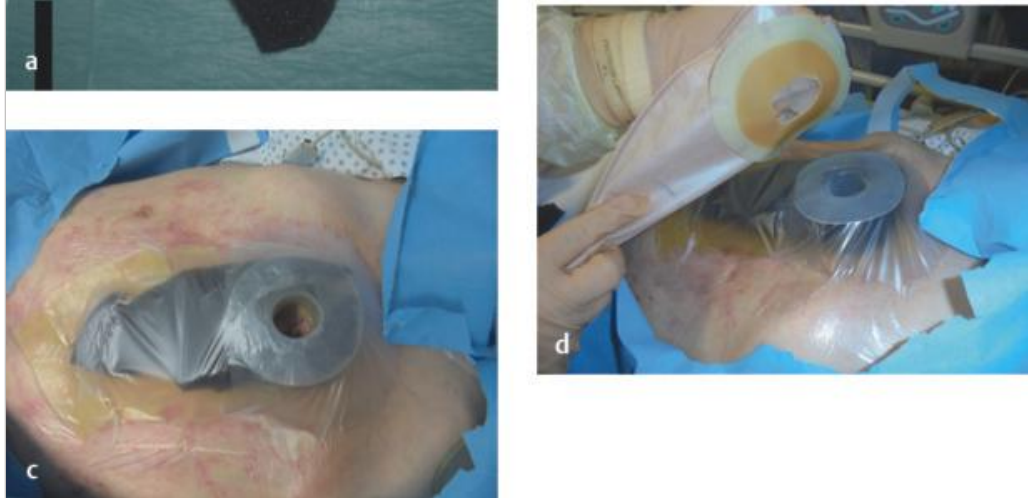

Abb.4.19 Versorgung mit Fisteladapter. (Quelle: Mit freundlicher Genehmigung von Phametra)

a Einpassen von Fisteladaptern (Beispiel), hier von unten gezeigt.

b Einpassen des Schaums mit integriertem Fisteladapter. Wundrandschutz und Abdichtung durch Comfeel zum Unterbauch hin.

c Abkleben des Unterdruckverbands mit Folie und Eröffnung über dem Fisteladapter.

d Anbringen des Ablaufbeutels über der Fistel. 


\section{Versorgung mit Wunddrainagebeuteln}

Großvolumige Wunddrainagebeutel von Eakin ( $\triangleright$ Abb. 4.20), ursprünglich zur Versorgung bei Anus praeter vorgesehen, eignen sich auch sehr gut zur Versorgung von postoperativen Wunden, Platzbäuchen, Stomata und Fisteln mit starker Exsudation. Diese Behandlung ermöglicht häufig eine frühzeitige Beendigung der NPWT trotz offenem Abdomen mit oder ohne Fistel.

Bei einer vorhandenen Fistel kann man diese zusätzlich mittels Hautschutzringen von der Wunde separieren. Die Wunde selbst kann dann mit Wundauflagen zur Abheilung gebracht werden und die Fistel stört dann nicht dabei. Lange Tragezeit von 4-6 Tagen.

\section{Cave}

Bei Entlassung ist zwingend eine Anlernphase des weiterversorgenden Pflegedienstes erforderlich.
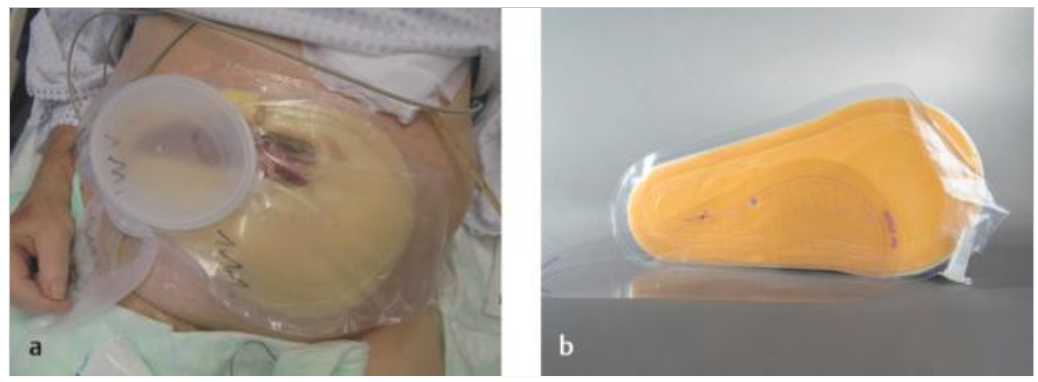

Abb. 4.20 Eakin Wundbeutel. a, b 


\subsection{Wunden bei Tumorpatienten (Palliativsituation)}

Maligne Läsionen der Haut sind für Patienten und Behandler eine oft schwer zu meisternde Situation. Diese Wunden erscheinen meist erst im präfinalen oder finalen Krankheitsstadium. Die meisten malignen Ulzera sind primäre Hauttumoren oder Hautmetastasen eines anderen Primärtumors - gefolgt von exulzerierenden Tumoren der Mamma, der Lunge, der Speicheldrüsen oder des Bauchraums, die aus tieferen Gewebeschichten durchbrechen ( $\triangleright$ Abb. 4.21, - Abb. 4.22).

Die nachfolgende Darstellung der Behandlung orientiert sich an den Leitlinien der deutschen Gesellschaft für Palliativmedizin (DGP), Sektion Pflege: Exulzerierende Wunden.

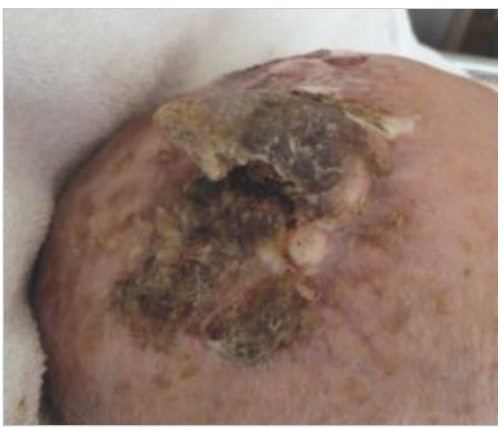

Abb.4.21 Tumorwunde Capillitium.

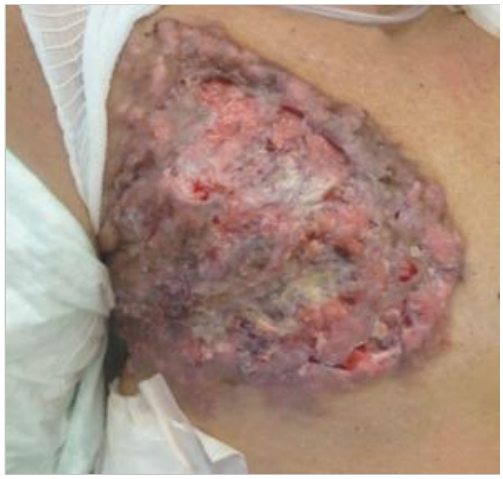

Abb.4.22 Tumorwunde Brust. 


\subsubsection{Ziele der Therapie}

- Eingehen auf persönliche Wünsche des Patienten (soweit medizinisch vertretbar)

- Exsudatmanagement

- Geruchslinderung

- atraumatische Verbandwechsel: Blutungen vermeiden und falls möglich Blutstillung

- Schutz des Wundrands/der Wundumgebung

- Auswahl kosmetisch akzeptabler Wundauflagen

- Verhinderung/Behandlung von Infektionen

- Linderung wundbezogener Schmerzen.

\subsubsection{Möglichkeiten der Lokalbehandlung wundbezogener Probleme}

Primäres Ziel ist es, situationsgerecht den Alltag des Patienten in möglichst vielen Lebensbereichen zu erleichtern. Therapeutische Ziele sind hier sekundär. Eine angemessene Patienten- und Angehörigen-Edukation ist unerlässlich.

\section{Exsudatmanagement}

- Die Standardversorgung bei mäßiger Exsudation ist die Versorgung mit Polyurethanschaumstoffen.

- Bei stärkerer Exsudation werden Produkte mit Superabsorber (auch als Saugkompressen) empfohlen.

- Höhlen können mit Alginaten, Hydrofasern oder Cavity-Produkten aufgefüllt werden.

- Für die Versorgung von Fisteln eignen sich Wunddrainagen, Stoma- oder Wunddrainage-Beutel.

- Eine Fixierung der Platte auf der Wunde ist oft erschwert. 


\section{Geruchsbekämpfung}

- Die Geruchsbildung erfolgt durch die bakterielle Besiedelung und in noch viel stärkerem Maße durch den Eiweißzerfall mit der Freisetzung von Putrescinen und Kadaverinen.

- Stark riechende Wunden/Tumoren können zur Geruchsminderung mit Leitungswasser ausgeduscht werden. Dabei ist auf die Verwendung von Sterilfiltern (s. Kap. 1.5.7) zu achten.

- Als Antiseptika bewähren sich Produkte auf Polyhexanid- oder OctenidinBasis.

- Octenidin darf nicht unter Druck in subkutanes Gewebe oder in Körperhöhlen eingebracht werden.

- Silberhaltige Wundauflagen reduzieren die Keimlast und damit häufig auch den Geruch.

- Bei anaeroben Keimen kann die systemische oder lokale (als Gel) Anwendung von Metronidazol hilfreich sein.

- Die Reduktion der Keimlast kann auch durch Produkte mit hydrophober Wechselwirkung oder mit Honigauflagen erzielt werden.

- Honig bindet zusätzlich noch Gerüche.

- Die stärkste Geruchsbindung erfolgt durch Kohleprodukte. Kohle hat ein Verhältnis der äußeren Oberfläche zur inneren Oberfläche von $1: 10000$, d.h. $4 \mathrm{~g}$ Aktivkohle haben die innere Fläche eines Fußballfeldes. Wichtig ist dabei, dass die Aktivkohleauflagen die Wunde komplett abdecken müssen.

- Ergänzend kann die topische Applikation von Chlorophyll-Lösung erfolgen (Chlorophyll wasserlöslich 2,5 g + Aqua cons. ad 100,0 g; konserviert mit Nipagin und Nipasol). Zur oralen Einnahme Stozzon-Dragees möglich.

- Geruchsneutralisation im Raum kann auch durch Aufstellen von Schalen mit Kaffeepulver, Essigwasser, Katzenstreu oder Rasierschaum erfolgen. Als künstlicher Geruchsbinder werden Nilodor-Tropfen angeboten. Die Raumaromatisierung mit Gegengerüchen wie ätherischen Ölen ist nur bedingt hilfreich.

- Auf den täglichen Wechsel der Körper- und Bettwäsche sowie ausreichende Raumlüftung ist zu achten. Für die Teilnahme am öffentlichen Leben kann die Wunde temporär (nur wenige Stunden!) mit einer Frischhaltefolie abgedeckt werden. 


\section{Wundrandschutz}

Auf den Schutz des Wundrandes ist wie bei allen chronischen Wunden zu achten, denn dadurch wird eine Wundvergrößerung verhindert (s. Kap. 5.1).

\section{Blutungen}

- Primär ulzerierte oder sekundär offen metastasierte Tumoren bluten extrem schnell! Daher ist unbedingt auf atraumatische Verbandwechsel mit nicht verklebenden Wundauflagen zu achten.

- Es empfiehlt sich, Materialien für den Fall einer Blutung bereitzuhalten, z. B.:

- Alginatkompressen für leichte Blutungen

- Eiswürfel oder Kühlelemente zum Auflegen

o dunkle Tücher.

- Bei stärkeren Blutungen können mit Xylometazolin (z. B. Otriven) oder Naphazolinnitrat (Privin) getränkte Kompressen verwendet werden.

- Auch Suprarenin in der Verdünnung 1:10 000, Tranexamsäure lokal oder systemisch oder resorbierbare Hämostyptika (z. B. Tabotamb, Gelita Tampon, Spongostan Standard) können ggf. verwendet werden. Als nicht resorbierbare Hämostyptika stehen Clauden Gaze oder Clauden Tupfer zur Verfügung. 